\title{
Tribological interaction between polytetrafluoroethylene and silicon oxide surfaces
}

A. Uçar, M. çopuroğlu, M. Z. Baykara, O. Arıkan, and S. Suzer

Citation: The Journal of Chemical Physics 141, 164702 (2014);

View online: https://doi.org/10.1063/1.4898384

View Table of Contents: http://aip.scitation.org/toc/jcp/141/16

Published by the American Institute of Physics

\section{Articles you may be interested in}

Artifacts related to tip asymmetry in high-resolution atomic force microscopy and scanning tunneling microscopy measurements of graphitic surfaces

Journal of Vacuum Science \& Technology B, Nanotechnology and Microelectronics: Materials, Processing, Measurement, and Phenomena 33, 031802 (2015); 10.1116/1.4915898

Effect of lateral tip stiffness on atomic-resolution force field spectroscopy

Journal of Vacuum Science \& Technology B, Nanotechnology and Microelectronics: Materials, Processing, Measurement, and Phenomena 31, 041801 (2013); 10.1116/1.4807376

Three-dimensional interaction force and tunneling current spectroscopy of point defects on rutile $\mathrm{TiO}_{2}(110)$ Applied Physics Letters 108, 071601 (2016); 10.1063/1.4942100

Combined low-temperature scanning tunneling/atomic force microscope for atomic resolution imaging and sitespecific force spectroscopy

Review of Scientific Instruments 79, 033704 (2008); 10.1063/1.2842631

Image contrast mechanisms in dynamic friction force microscopy: Antimony particles on graphite Journal of Applied Physics 121, 044307 (2017); 10.1063/1.4974882

Self-forming oriented layer slip and macroscale super-low friction of graphene Applied Physics Letters 110, 073101 (2017); 10.1063/1.4975979

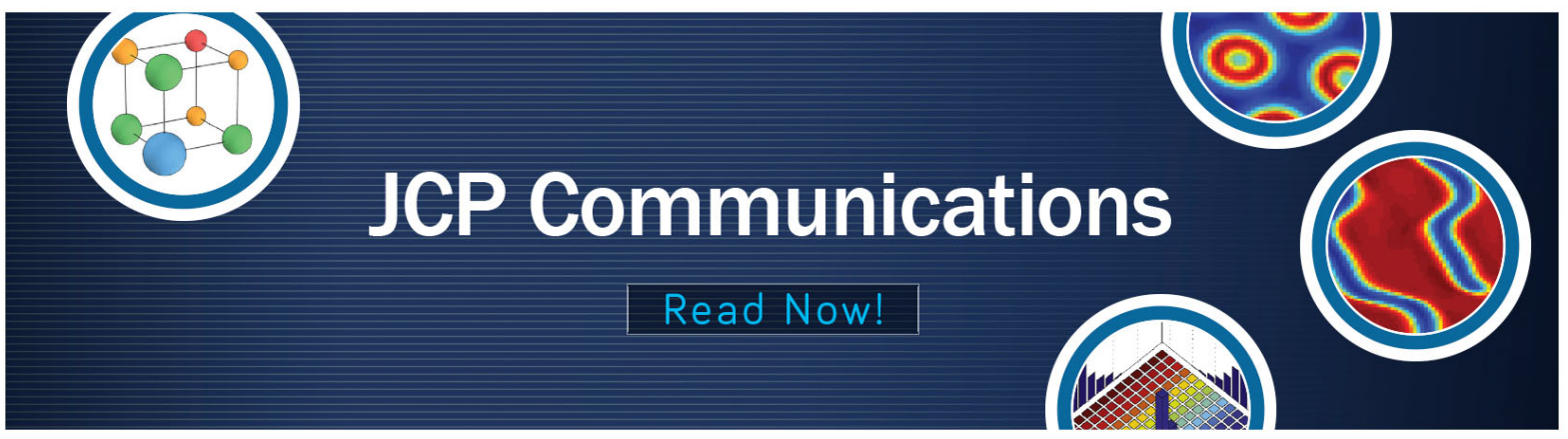




\title{
Tribological interaction between polytetrafluoroethylene and silicon oxide surfaces
}

\author{
A. Uçar, ${ }^{1}$ M. Çopuroğlu, ${ }^{1}$ M. Z. Baykara, ${ }^{2}$ O. Arıkan, ${ }^{3}$ and S. Suzer ${ }^{1, a)}$ \\ ${ }^{1}$ Department of Chemistry, Bilkent University, 06800 Ankara, Turkey \\ ${ }^{2}$ Department of Mechanical Engineering, Bilkent University, 06800 Ankara, Turkey \\ ${ }^{3}$ Department of Electrical and Electronics Engineering, Bilkent University, 06800 Ankara, Turkey
}

(Received 29 July 2014; accepted 6 October 2014; published online 23 October 2014)

\begin{abstract}
We investigated the tribological interaction between polytetrafluoroethylene (PTFE) and silicon oxide surfaces. A simple rig was designed to bring about a friction between the surfaces via sliding a piece of PTFE on a thermally oxidized silicon wafer specimen. A very mild inclination $\left(\sim 0.5^{\circ}\right)$ along the sliding motion was also employed in order to monitor the tribological interaction in a gradual manner as a function of increasing contact force. Additionally, some patterns were sketched on the silicon oxide surface using the PTFE tip to investigate changes produced in the hydrophobicity of the surface, where the approximate water contact angle was $45^{\circ}$ before the transfer. The nature of the transferred materials was characterized by X-ray photoelectron spectroscopy (XPS) and scanning electron microscopy (SEM). XPS results revealed that PTFE was faithfully transferred onto the silicon oxide surface upon even at the slightest contact and SEM images demonstrated that stable morphological changes could be imparted onto the surface. The minimum apparent contact pressure to realize the PTFE transfer is estimated as $5 \mathrm{kPa}$, much lower than reported previously. Stability of the patterns imparted towards many chemical washing processes lead us to postulate that the interaction is most likely to be chemical. Contact angle measurements, which were carried out to characterize and monitor the hydrophobicity of the silicon oxide surface, showed that upon PTFE transfer the hydrophobicity of the $\mathrm{SiO}_{2}$ surface could be significantly enhanced, which might also depend upon the pattern sketched onto the surface. Contact angle values above $100^{\circ}$ were obtained. (c) 2014 AIP Publishing LLC. [http://dx.doi.org/10.1063/1.4898384]
\end{abstract}

\section{INTRODUCTION}

Tribology generally refers to physical/chemical reactions/interactions which proceed between two contacting surfaces. ${ }^{1}$ Identification of the physical and chemical nature of these interactions has been of great academic as well as commercial importance for more than 5 decades. ${ }^{1-12}$ Makinson and Tabor studied the transfer and friction of polytetrafluoroethylene (PTFE) sliding on glass using optical and scanning electron microscopic techniques, where they demonstrated that PTFE could be transferred onto other surfaces during sliding motion. ${ }^{1}$ They also identified two frictional regimes (low and high) depending on both the temperature at which the sliding was carried out and the speed of the sliding motion. In the low-friction regime (at low sliding speed and/or high temperatures) it was observed that a PTFE film whose thickness varied between 10 and $40 \mathrm{~nm}$ was transferred onto the glass surface. This study was then extended to other thermoplastic polymers such as commercial copolymer of fluorinated ethylene propylene, polychlorotrifluoroethylene (PCTFE), polyethylene (PE), polystyrene (PS), poly(methylmethacrylate) (PMMA), and polyvinylchloride (PVC), where tribological transfer of those polymers onto clean surfaces was described. ${ }^{2}$ In another study, Pepper examined the interaction of PTFE, PCTFE and PVC with various

\footnotetext{
a) suzer@fen.bilkent.edu.tr
}

surfaces by Auger electron spectroscopy. ${ }^{3}$ The work showed that sliding PTFE over metal surfaces would result in formation of a continuous uniform PTFE film, which was about 2-4 atomic layer-thick. However, for PVC film transfer was not observed as a result of sliding; instead chemisorbed chlorine was observed, and PCTFE produced chemisorbed chain fragments on the metal surface. X-ray photoelectron spectroscopy (XPS) was also utilized to study the tribological interaction of various metals and metal oxides with PTFE. ${ }^{4,5}$ Wittmann and Smith demonstrated that some materials grown on PTFE that was tribologically deposited on a glass surface would exhibit significantly higher levels of alignment and this could be used as a new technique to produce highly oriented thin films. ${ }^{6}$ Others have extended such studies by frictional transferring of PTFE by sliding a bar of Teflon onto various material surfaces, including metals. For obtaining highly oriented PTFE structures, they have investigated the effects of temperature in the range of $0-300^{\circ} \mathrm{C}$, the load applied and the sliding speed, and established that optimum; (i) temperatures are above $150^{\circ} \mathrm{C}$, at which Teflon softens, and (ii) pressures are within the $0.1-10 \mathrm{MPa}$, and (iii) sliding speeds are in the range of $0.1-10 \mathrm{~mm} / \mathrm{s}^{7-10}$

Although many other studies on PTFE transfer to another solid surface have been published, most have focused on improvement of the physical properties of surfaces, i.e., achieving lower friction coefficients and wear rates. ${ }^{1-19}$ However, the nature of those interactions at the atomic scale has not 
been completely understood. To this end, applying computational chemistry methods as a tool for analyzing the mechanism of friction and transfer film formation has recently been reported. For instance, using methods based on density functional theory, quantum chemical molecular dynamics, and classical molecular dynamics, Onedera et al. showed that the ionic bonds occurring at the interfaces of PTFE and aluminum oxide could play a significant role in the formation of the PTFE film on the aluminum oxide surface. ${ }^{20}$ In that study, they also illustrated the influence of certain environmental conditions on the transfer film formation and found that the extent of PTFE transfer to aluminum oxide surface in nitrogen gas was less than that in water vapor, since the interacting nitrogen molecules would shield the formation of ionic bonds at the sliding interfaces. Considering the wide range of use of PTFE for numerous applications, including biomedical devices and tools ${ }^{21}$ further understanding of the interactions between solid surfaces such as polymers and inorganic substances is undoubtedly of high scientific and commercial importance.

In addition to its wide range of use in tribological studies, PTFE has also been used to alter the wetting characteristics of surfaces due to its low surface energy. Enhancing the hydrophobicity of surfaces using PTFE, for instance, has attracted a great deal of interest in a number of studies. ${ }^{22-25}$ Therefore developing new methods for transferring PTFE onto various surfaces in a tribological way in order to tune the surface wettability is also of potential importance.

The purpose of this study is to investigate the physi$\mathrm{cal} /$ chemical nature of the interaction between a PTFE tip and thermally oxidized silicon in contact and to develop a new tribological method for tuning the wettability of silicon oxide surfaces by PTFE transfer. To achieve this goal, a simple rig was set up to bring about a frictional contact between the surfaces via sliding a PTFE tip on a thermally oxidized silicon wafer specimen where the measured water contact angle was $45^{\circ}$ before the application. An optional, small $\left(\sim 0.5^{\circ}\right)$ inclination along the sliding motion was also employed such that the contact force arising between the PTFE tip and the silicon oxide surface gradually increased during sliding. In this way, the tribological interaction between PTFE and silicon oxide can be monitored as a function of contact force in a gradual manner. Additionally, certain patterns were sketched on the silicon oxide surface using the PTFE tip to investigate and control the hydrophobicity of the surface. X-ray photoelectron spectroscopy (XPS), a surface sensitive analysis technique with high chemical selectivity, ${ }^{26-28}$ was utilized as the main tool for the verification and further analysis of the PTFE transfer. The fact that PTFE is a halogenated polymer provides an analytically simpler identification of the transfer characteristics by evaluating $\mathrm{F} 1 \mathrm{~s}$ and $\mathrm{C} 1 \mathrm{~s}$ peaks in a more detailed manner. Moreover, as mentioned before, gradually enhanced tribological interaction that was brought about by employing the inclined surface along the motion was investigated. Potential changes rendered on the silicon oxide surface morphology upon sliding contact were examined by Scanning Electron Microscopy (SEM). For assessment of hydrophobicity/wettability of the surfaces, water contact angle measurements were carried out. Silicon/silicon oxide substrates were chosen since they provide very smooth surfaces, and also for ease of modification of their acid-base character by aqueous solutions. ${ }^{16}$ It is expected the results can also be extended to other similar and technologically important substrates like glass, quartz, $\mathrm{Al}_{2} \mathrm{O}_{3}, \mathrm{TiO}_{2}, \mathrm{Si}_{3} \mathrm{~N}_{4}$, TiN, ITO, etc., for other applications.

\section{EXPERIMENTAL}

Commercially available n-type $\operatorname{Si}(100)$ wafers with a conductivity of $\sim 20 \Omega$-cm and doped with $\mathrm{P}$ were obtained from the Institute of Electronic Materials Technology (ITME). The wafers were cleaved into $1-2 \mathrm{~cm}^{2}$-pieces. They were then cleaned with concentrated aqueous HF solution for $\sim 30 \mathrm{~s}$ to remove the native oxide layer and washed with deionized water and dried. This step was repeated three times. Following this procedure, $\sim 10 \mathrm{~nm}$-thick oxide layers were grown by annealing the wafer specimens in air at $700^{\circ} \mathrm{C}$ for $3 \mathrm{~h}$. Afterwards, all samples were immersed in deionized water for $1 \mathrm{~h}$. The water used in all experiments was obtained from a three-stage Millipore Milli-Q Synergy 185 purification system. The PTFE tip employed in the sliding experiments was cut out from a larger general-purpose PTFE sheet with a thickness of $1 \mathrm{~mm}$. An X-Y-Z stage (Nanomagnetics) that allowed micrometer-scale-motion in 2D was used to slide the tip of the PTFE over the silicon oxide surface. As mentioned before, an optional, very small inclination $\left(\sim 0.5^{\circ}\right)$ along the sliding motion was also employed in order to monitor the tribological interaction in a gradual manner as a function of increasing contact force, as depicted in Fig. 1. In this figure, the optical and electron microscope images of the PTFE tip used in the study are also presented (upper left and upper right of the figure, respectively), which are employed to gain
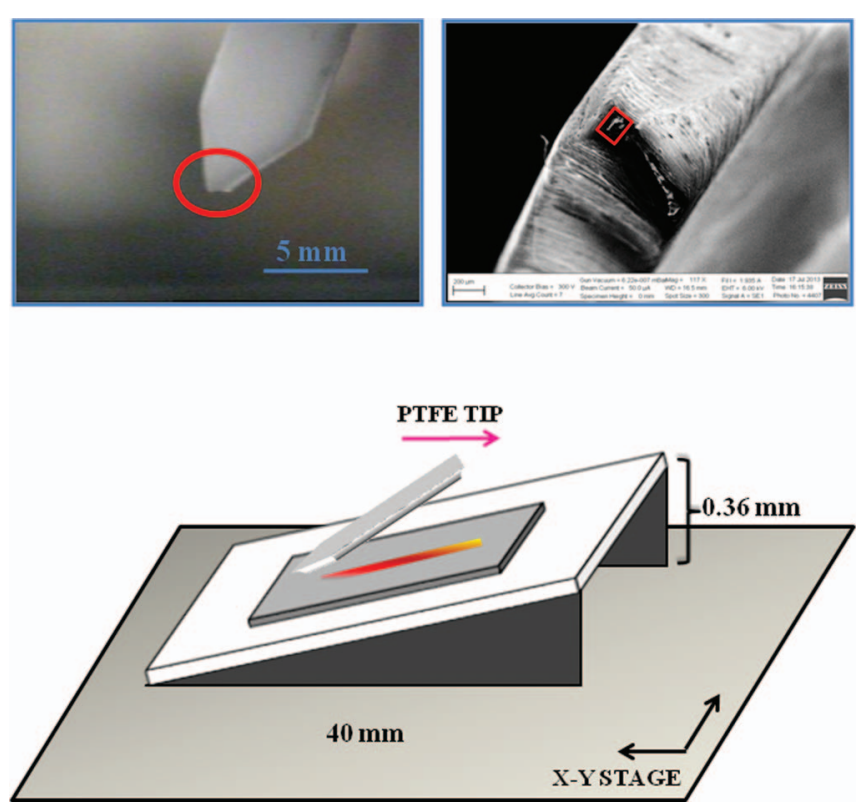

FIG. 1. A schematic representation of the rig used to bring about gradual tribological interaction between PTFE tip and silicon oxide surface. Optical and electron microscope images (upper left and right, respectively) of the PTFE tip are also presented. The apex of the tip is highlighted with a red rectangle. 
information about the dimensions of the apparent contact area between the PTFE tip and the wafer surface. The drawing of individual scratch lines in our experiments is performed with a speed of about $1 \mathrm{~mm} / \mathrm{s}(0.001 \mathrm{~m} / \mathrm{s})$ and all experiments were performed under ambient laboratory conditions.

A Thermo Fisher K-alpha electron spectrometer with a monochromatic AlK $\alpha$ X-ray source $(\mathrm{h} v=1486.6 \mathrm{eV})$ was used for the surface chemical analysis of the specimens. Changes rendered on the silicon oxide surface morphology were examined using a Zeiss Evo 40 Scanning Electron Microscope. A Tantec CAM-Micro Contact Angle Meter was used for the assessment of surface hydrophobicity/wettability.

\section{RESULTS AND DISCUSSION}

\section{A. XPS analyses}

The upper image in Fig. 2 displays an aerial XPS spectral map of the F1s peak area, recorded in the snap-shot mode of the instrument, performed on the surface of the sample where some lines drawn by the PTFE tip are located. These data were taken by $30 \mu \mathrm{m}$-diameter X-ray spot size and with steps of $30 \mu \mathrm{m}$ between the data points. A detailed normal scanned $\mathrm{F} 1 \mathrm{~s}$ and $\mathrm{C} 1 \mathrm{~s}$ regions on one of the points on the scratched line is also shown and clearly demonstrates that not only fluorine but complete PTFE transfer has been realized on the silicon oxide surface. This was verified by both the position and the intensity ratio of the $\mathrm{C} 1 \mathrm{~s}(\sim 294 \mathrm{eV})$, and F1s $(\sim 691 \mathrm{eV})$ peaks, which are assigned to $-\mathrm{CF}_{2}-$, after charge correction. The measured ratio of $\mathrm{F}: \mathrm{C}(\sim 2)$ also reflects the stoichiometry of the transferred species. ${ }^{27}$

We now ask the following relevant questions and try to answer them consequently: (i) Do PTFE segments transfer faithfully or can we detect decomposition leading to; for example, Si-F bond formation or other fluorinated and chemisorbed moieties? (ii) What are the contact forces (and related apparent-area contact pressures) associated with this PTFE transfer? In order to answer both, we analyzed samples scratched using the setup shown in Figure 1, starting with the tip not touching the silicon oxide surface placed at the angle designated. As the PTFE tip is gradually moved towards the inclined wafer surface, there will be a point at which the tip starts interacting with the surface. Figure 3 displays the areal map of the F1s peak, recorded in the snapshot mode. We then go back and perform line-scan analysis along the designated line by recording individual scans of $\mathrm{F} 1 \mathrm{~s}, \mathrm{C} 1 \mathrm{~s}$, and $\mathrm{O} 1 \mathrm{~s}$ regions, acquired using an X-ray spot size of $70 \mu \mathrm{m}$ with step sizes of $70 \mu \mathrm{m}$. As seen from the Figs. 3(b) and 3(c), the extent of PTFE transfer gradually increases along the movement on the inclined surface accompanied by a slight decrease in the intensity of the O1s peak (Fig. 3(d)), both verifying PTFE transfer onto the silicon oxide surface. In Fig. 3(e), results of analysis conducted for reliable identification of the fitted peaks to $\mathrm{F} 1 \mathrm{~s}, \mathrm{C} 1 \mathrm{~s}$ (both the adventitious and the $\mathrm{CF}_{2}$ carbons) and $\mathrm{O} 1 \mathrm{~s}$ regions are also shown. In order to identify the onset position, peak areas of $\mathrm{C} 1 \mathrm{~s}$ that are within or below the extracted noise level are set to zero. As can be seen, the obtained onset position on $\mathrm{C} 1 \mathrm{~s}$ also correlates well with the analysis of the fitted peaks to F1s and O1s.

From the numerous samples we have prepared and analyzed over the last 2 years, and within our measurement limits with XPS, we have always observed that entire PTFE segments are faithfully transferred to silicon oxide surfaces forming stable (up to 6 months and longer) features created by frictional sliding of the PTFE tip. Note in passing, that the position of the F1s peak is very sensitive to metal-fluoride, and/or, oxide-fluoride bonding which we have not observed. Perhaps more importantly, the individual spectra, which were derived from their respective line scans, and are presented at the bottom of Fig. 3 revealed that PTFE transfer could occur upon even at the slightest contact. SEM images also provide additional support for the evidence that the frictional sliding process could indeed impart stable PTFE segments on to the silicon oxide surfaces, as shown in Figure 4. Furthermore, the scratches imparted are stable even after several cleaning processes, as revealed by the XPS maps recorded afterwards,
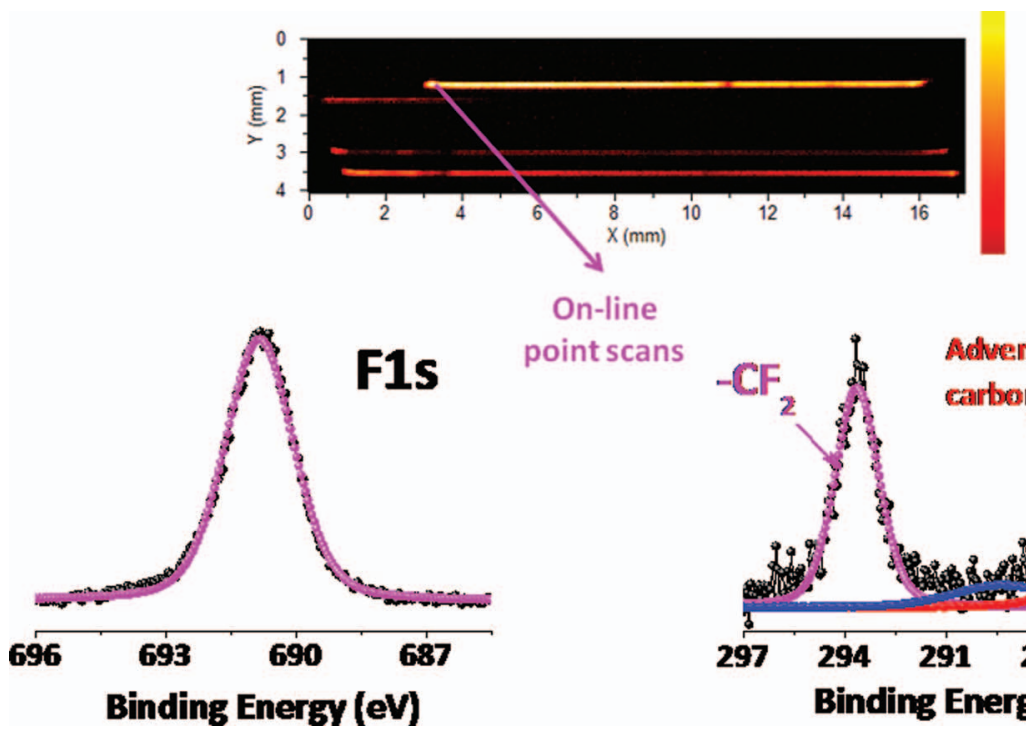

On-line point scans

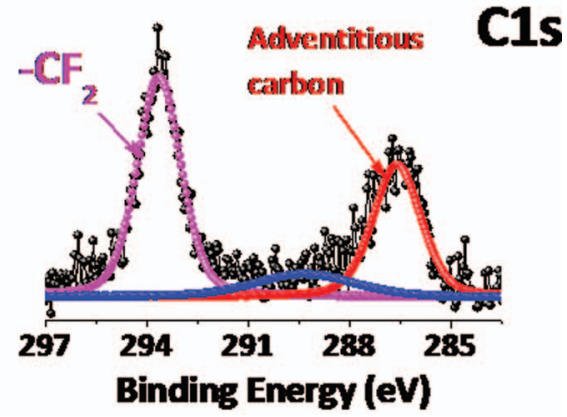

FIG. 2. An aerial spectral map of the F1s peak intensity, performed on the surface of the sample where some lines were drawn by the PTFE tip. F1s and C1s regions of XPS spectra recorded on a point on the line are also shown. 
(a)

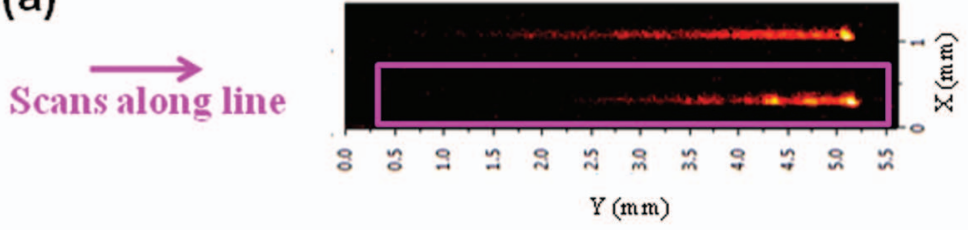

(b)
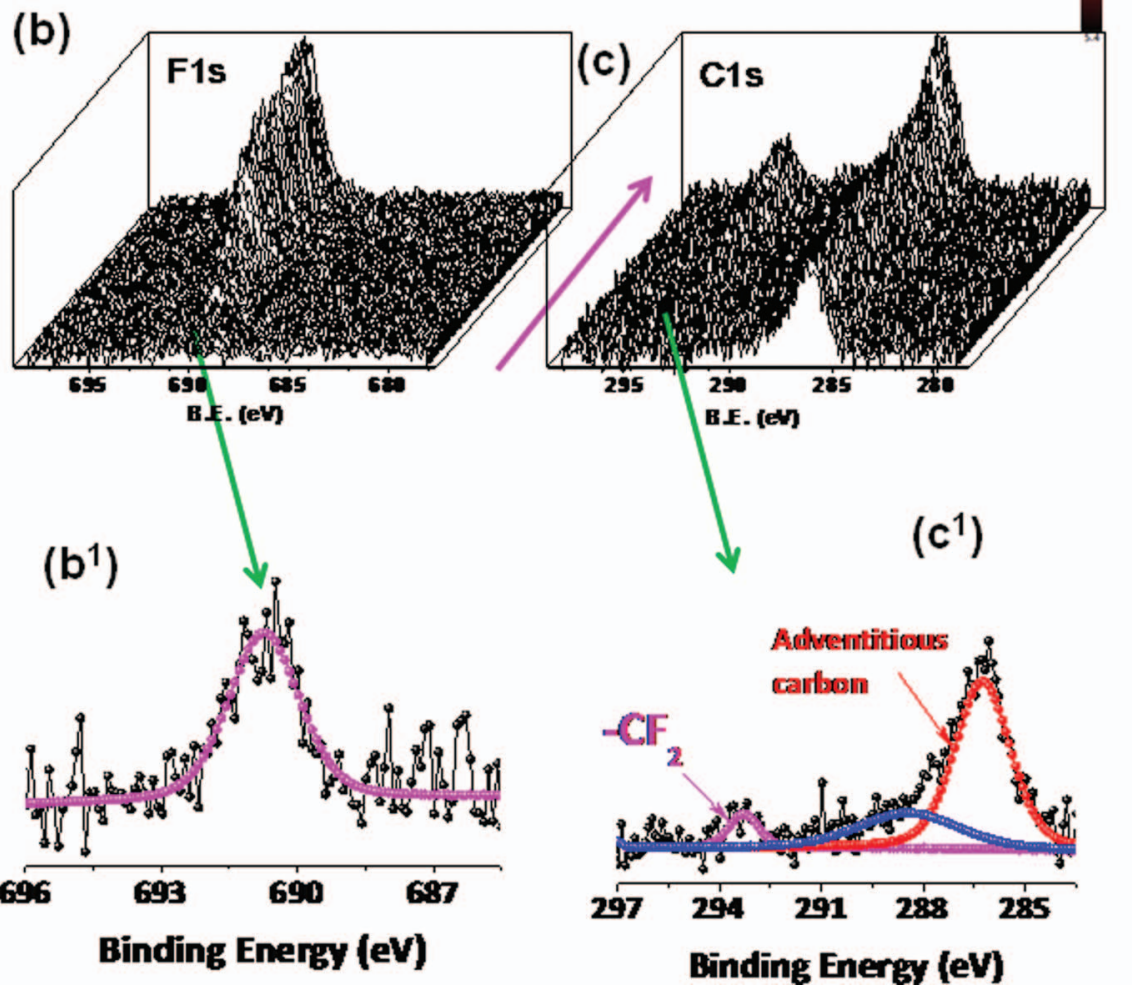

Area Profile

F1s

$(\mathrm{min})$

(d)
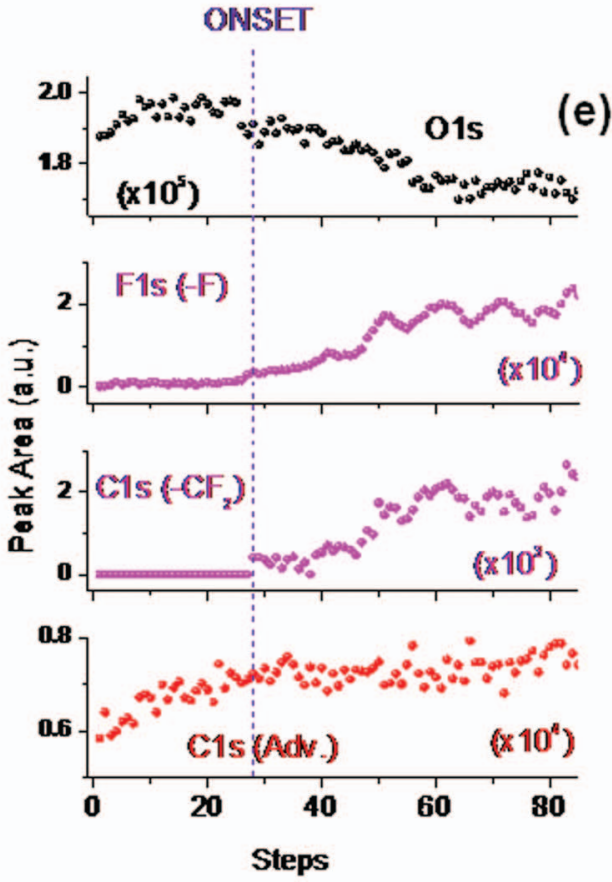

FIG. 3. (a) Gradual increase of PTFE transfer on the surface of the specimen and recorded line scan spectra; (b) F1s, (c) C1s, and (d) O1s regions. The spectra at the onset are also shown in $\left(\mathrm{b}^{1}\right)$ for the F1s and $\left(\mathrm{c}^{1}\right)$ for the $\mathrm{C} 1 \mathrm{~s}$ regions. (e) Displays the plots of the peaks' areas along the scanned line, from which the onset of the PTFE transfer is evaluated.

shown in Figure 5, which displays the F1s image recorded after the following steps: (i) Flashing the sample with flowing $\mathrm{N}_{2}$ gas. (ii) Washing with ethanol. (iii) Washing with acetone in ultrasonic bath for 15 minutes. The fact that the feature imprinted is stable after all these treatments with almost no loss in F1s intensity strongly supports our claim that the interaction is most likely to be chemical in nature.

Complementary to XPS measurements, the contact force and related apparent-area contact pressure arising between the PTFE tip and the silicon oxide surface during sliding experi-
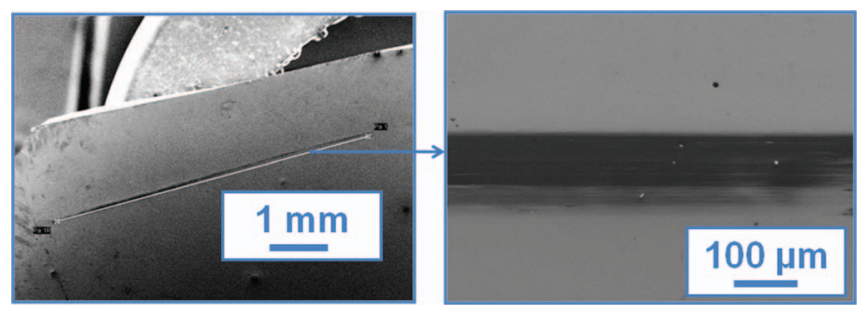

FIG. 4. Changes on the surface morphology upon PTFE transfer demonstrated by SEM. ments was estimated analytically, using the onset determined from Figure 3, in order to study the associated effects on tribological transfer of PTFE. Towards this end, the standard elastic beam deflection formula used to relate the transverse

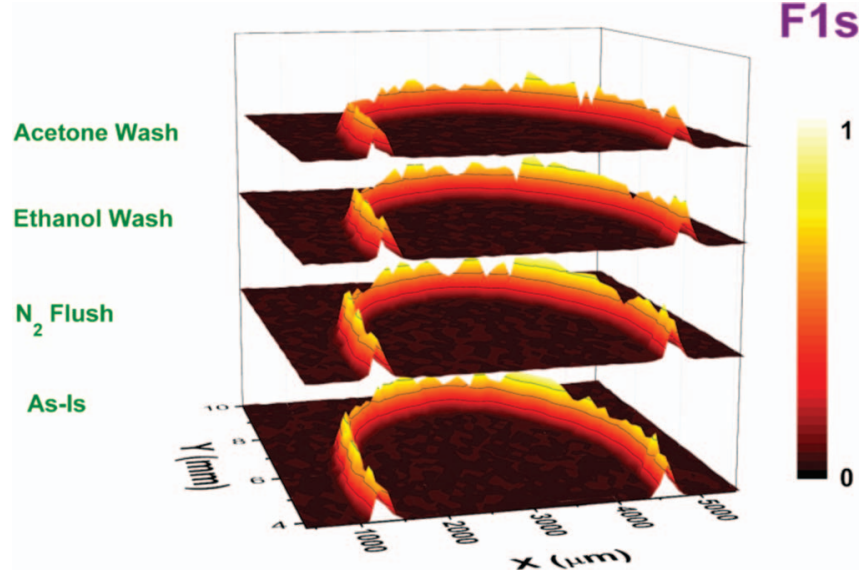

FIG. 5. F1s areal maps of a section of a circular scratch drawn, recorded after several physical and chemical washing steps. 
force applied at the free end of a cantilevered beam $(\mathbf{P})$ to the deflection undergone $(\delta)$ in the same direction has been used: 29

$$
\mathbf{P}=\left(\mathbf{3 E} * \mathbf{I} / \mathbf{L}^{\mathbf{3}}\right) * \boldsymbol{\delta},
$$

where $\mathbf{E}$ is the Young's Modulus of PTFE $(0.5 \mathrm{GPa}),{ }^{30}$ and $\mathbf{I}$ is the moment of inertia of the PTFE beam around the bending axis (determined by taking into account the thickness of the PTFE beam $(1 \mathrm{~mm})$ and its width $(5 \mathrm{~mm}))$, and $\mathbf{L}$ is the length of the beam $(37 \mathrm{~mm})$. Note that the sliding contact angle between the PTFE beam and the silicon oxide surface has also been taken into account during the calculation of the contact force between the tip and the surface. In our experimental setup, the tip of the beam is continuously pushed along an inclined $\mathrm{SiO}_{2}$ surface, thus the associated transverse deflection at the tip and consequently, the contact force acting between the Teflon beam and the $\mathrm{SiO}_{2}$ surface increase gradually. In this way, it becomes possible to observe the effect of increasing contact forces (and apparent pressures) on tribological PTFE transfer using individual scratches on the surface. The small amount of transverse deflection undergone by the beam during sliding $(<50 \mu \mathrm{m}$ for a typical line of $5 \mathrm{~mm}$ length) justifies the use of the elastic beam deflection formula and the deflection rate of $10 \mu \mathrm{m} / \mathrm{s}$ allows us to neglect viscoelastic and thermal effects on mechanical behavior.

Using the above-mentioned experimental parameters, the maximum contact force arising between the PTFE tip and the sample surface is estimated to be about $0.06 \mathrm{mN}$, at the onset of tribological transfer of PTFE. Studying the upper-right inset in Fig. 1 and the SEM image of a typical scratch line in Fig. 4, the region of contact between the PTFE tip and the silicon oxide surface can be estimated to be approximately $100 \mu \mathrm{m} \times 125 \mu \mathrm{m}$, leading to a maximum contact pressure of about $5 \mathrm{kPa}$ at the onset. It should be noted here that the accuracy in the estimation made regarding the contact area is limited by the actual, multi-asperity character of the contact interface and as such, the reported value of $5 \mathrm{kPa}$ des- ignates an "apparent-area contact pressure," in accordance with common practice in tribological studies performed using tribometer-based approaches. ${ }^{31,32}$ Considering that similar investigations reported in the literature using atomic force microscopy and tribometers have employed contact pressures in the 6-40 MPa range, our results remarkably reveal that tribological transfer of PTFE starts occurring at much smaller contact pressures, essentially at few $\mathrm{kPa}^{31-34}$ It is essential to note that the contact forces and associated pressures in our experimental setup are brought about by the tiny deflections $(<50 \mu \mathrm{m})$ of a soft spring in the form of the PTFE cantilever, while the pressures corresponding to traditional tribometerbased experiments employed in previous tribological studies on PTFE are obtained by the equivalent of at least tens of kilograms of load on a contact area on the order of $1 \mathrm{~cm}^{2}$.

We have also carried out measurements on the PTFE transfer to silicon oxide surfaces which have been treated with aqueous acidic and basic solutions before the transfer. Although such treatments were reported to cause drastic triboelectrical changes of the silicon oxide surfaces, ${ }^{16}$ we were not able observe any significant difference between the acid or base treated and untreated samples. In light of these new findings, additional experiments are definitely needed to clarify and further our understanding about the nature of the forces contributing to the mechanism(s) of this material transfer both from the PTFE side and also related to the surface. Along this line, washing with stronger chemicals in terms of affecting the removal of the PTFE features imparted is one of our plans for future work.

\section{B. Tuning the hydrophobicity}

To control and tune the surface energy of the modified silicon surfaces, various features were imparted to create different stable morphologies. PTFE slide-transferred lines were drawn on the silicon oxide surface with different densities, determined by the number of lines drawn in $1 \mathrm{~mm}$ (for example,
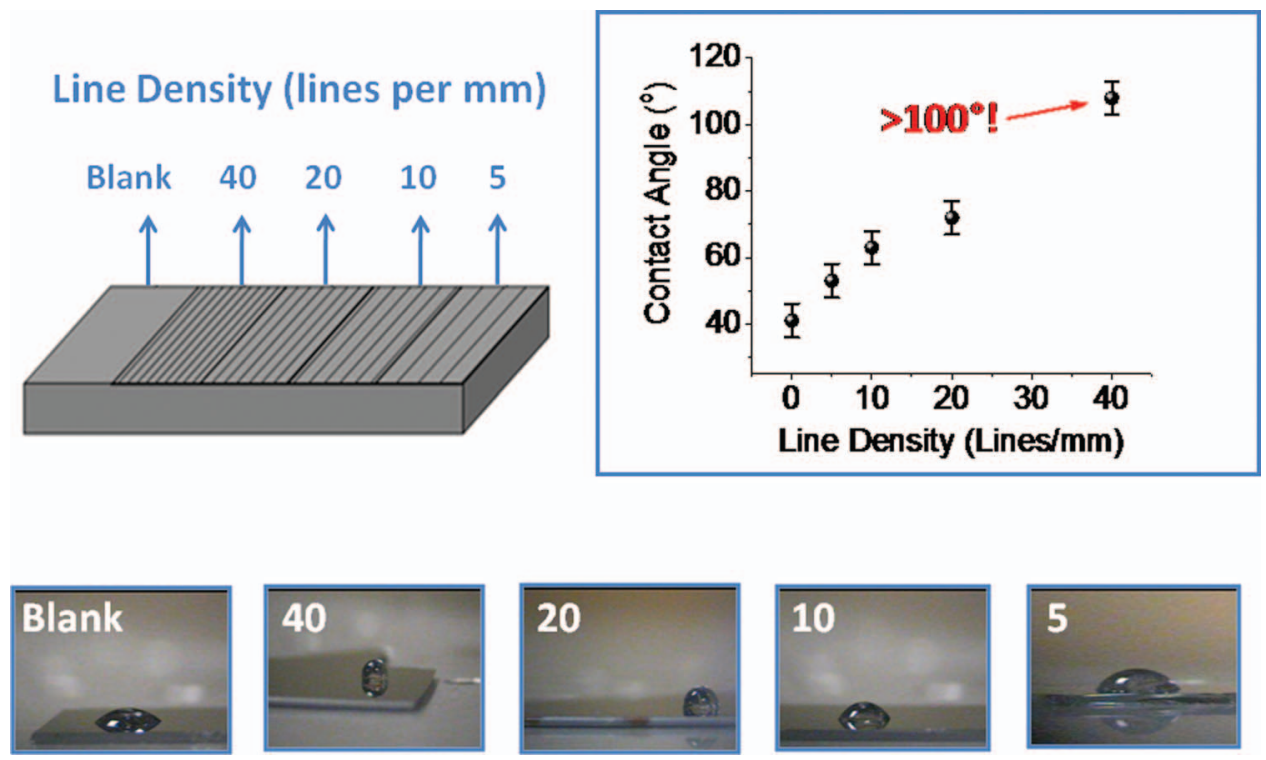

FIG. 6. Water contact angle measurement results for the surfaces with various PTFE line densities. Images of droplets taken by the optical microscope are also presented. 
lines drawn with $200 \mu \mathrm{m}$ intervals correspond to 5 lines $/ \mathrm{mm}$ ), and water contact measurements were carried out. The contact pressure applied in this case was less than 10 times of the minimum pressure needed as judged by the intensity of the F1s peak. As seen in Figure 6, a dramatic increase in the contact angle value was observed with line density and a value above $100^{\circ}$ was achieved in the case of 40 lines per $\mathrm{mm}$. This is the highest density, which is dictated by the capability of our present experimental setup.

\section{CONCLUSIONS}

In this work, the tribological interaction between PTFE and silicon oxide surfaces was investigated using XPS. It was demonstrated that PTFE could indeed be successfully transferred onto silicon oxide surfaces by drawing lines. Stability of the patterns produced even after many chemical washing processes lead us to postulate that the nature of the interaction is very likely to be chemical. Hydrophobicity was also shown to be tunable by PTFE patterns sketched on the surface, yielding Water-Contact-Angle values above $100^{\circ}$. It was also found that the minimum apparent-area contact pressure required to transfer PTFE onto the silicon oxide surface was less than $5 \mathrm{kPa}$, at room temperature, which is more than 2 orders of magnitude lower than reported before. Overall, the simple method described in this article used to study the tribological interaction of PTFE and silicon oxide surfaces would thus provide a new point of view with regards to tuning the wetting and characteristics of surfaces for numerous potential applications. ${ }^{35}$

\section{ACKNOWLEDGMENTS}

This work was partially supported by TÜBITAK, through the Grant No. 212 M 051. The authors would like to acknowledge Dr. Şakir Baytaroğlu of Department of Mechanical Engineering, Bilkent University, and NanoMagnetics Instruments Inc. for their assistance in designing and constructing the experimental setups.

${ }^{1}$ K. R. Makinson and D. Tabor, Proc. R. Soc. London, Ser. A 281, 49 (1964). ${ }^{2}$ C. M. Pooley and D. Tabor, Proc. R. Soc. London, Ser. A 329, 251 (1972).

${ }^{3}$ S. V. Pepper, J. Appl. Phys. 45, 2947 (1974).

${ }^{4}$ D. L. Gong, B. Zhang, Q. J. Xue, and H. L. Wang, J. Appl. Polym. Sci. 41, 2587 (1990)

${ }^{5}$ G. Deli, X. Qunji, and W. Hongli, Wear 148, 161 (1991).

${ }^{6}$ J. C. Wittmann and P. Smith, Nature (London) 352, 414 (1991).
${ }^{7}$ D. Fenwick, K. J. Ihn, F. Motamedi, J. C. Wittmann, and P. Smith, J. Appl. Polym. Sci. 50, 1151 (1993).

${ }^{8}$ P. Bodoe, C. Ziegler, J. R. Rasmusson, W. R. Salaneck, and D. T. Clark, Synth. Met. 55-57, 329 (1993).

${ }^{9}$ P. Bodö and M. Schott, Thin Solid Films 286, 98 (1996).

${ }^{10}$ D. W. Breiby, T. I. Sølling, O. Bunk, R. B. Nyberg, K. Norrman, and M. M. Nielsen, Macromolecules 38, 2383 (2005).

${ }^{11}$ T. Blanchet and F. Kennedy, Wear 153, 229 (1992).

${ }^{12}$ G. Beamson, D. T. Clark, D. E. Deegan, N. W. Hayes, D. S. L. Law, J. R. Rasmusson, and W. R. Salaneck, Surf. Interface Anal. 24, 204 (1996).

${ }^{13}$ S. M. Hsu and R. S. Gates, J. Phys. D: Appl. Phys. 39, 3128 (2006).

${ }^{14}$ X. Lu, K. Wong, P. Wong, K. Mitchell, J. Cotter, and D. Eadie, Wear 261, 1155 (2006).

${ }^{15}$ J. Yu, S. H. Kim, B. Yu, L. Qian, and Z. Zhou, ACS Appl. Mater. Interfaces 4, 1585 (2012).

${ }^{16}$ J. J. Cole, C. R. Barry, R. J. Knuesel, X. Wang, and H. O. Jacobs, Langmuir 27, 7321 (2011).

${ }^{17}$ H. T. Baytekin, B. Baytekin, J. T. Incorvati, and B. A. Grzybowski, Angew. Chem., Int. Ed. 124, 4927 (2012).

${ }^{18}$ C. Gabler, E. Pittenauer, N. Dörr, and G. Allmaier, Anal. Chem. 84, 10708 (2012).

${ }^{19}$ J. D. Schall, G. Gao, and J. A. Harrison, J. Phys. Chem. C 114, 5321 (2010).

${ }^{20}$ T. Onodera, M. Park, K. Souma, N. Ozawa, and M. Kubo, J. Phys. Chem. C 117, 10464 (2013).

${ }^{21}$ C. Gehrke, H. Li, and H. Sant, Biomed. Microdevices 16, 173 (2014).

${ }^{22}$ K. K. S. Lau, J. Bico, K. B. K. Teo, M. Chhowalla, G. A. J. Amaratunga, W. I. Milne, G. H. Mckinley, and K. K. Gleason, Nano Lett. 3, 1701 (2003).

${ }^{23}$ L. Feng, Z. Zhang, Z. Mai, Y. Ma, B. Liu, L. Jiang, and D. Zhu, Angew. Chem., Int. Ed. 43, 2012 (2004).

${ }^{24}$ W. Hou and Q. J. Wang, J. Colloid Interface Sci. 333, 400 (2009).

${ }^{25}$ T. Kamegawa, Y. Shimizu, and H. Yamashita, Adv. Mater. 24, 3697 (2012).

${ }^{26}$ D. Briggs and M. P. Seah, Practical Surface Analysis: Auger and X-ray Photoelectron Spectroscopy (Wiley, New York, 1983).

${ }^{27}$ G. Beamson and D. Briggs, High Resolution XPS of Organic Polymers: the Scienta ESCA300 Database (Wiley, Chichester, 1992).

${ }^{28} \mathrm{H}$. Sezen and S. Suzer, Thin Solid Films 534, 1 (2013).

${ }^{29}$ R. C. Hibbeler, Mechanics of Materials, 9 th ed. (Prentice Hall, Upper Saddle River, 2013).

${ }^{30}$ Mechanical testing and evaluation Vol. 8, ASM Handbook, Vol. 10th ed., edited by H. Kuhn and D. Medlin (ASM International, Materials Park, OH, 2000).

${ }^{31}$ I. Jang, D. L. Burris, P. L. Dickrell, P. R. Barry, C. Santos, S. S. Perry, S. R. Phillpot, S. B. Sinnott, and G. W. Sawyer, J. Appl. Phys. 102, 123509 (2007).

${ }^{32}$ N. L. McCook, D. L. Burris, P. L. Dickrell, and W. G. Sawyer, Tribol. Lett. 20, 109 (2005).

${ }^{33}$ I. Tzanakis, M. Conte, M. Hadfield, and T. A. Stolarski, Wear 303, 154 (2013).

${ }^{34}$ J. T. Shen, Y. T. Pei, and J. Th. M. De Hosson, J. Mater. Sci. 49, 1484 (2014).

${ }^{35}$ See supplementary material at http://dx.doi.org/10.1063/1.4898384 for a video file of the XPS data in the snapshot mode collected from a silicon oxide surface, onto which PTFE was tribologically transferred in the pattern "biluni" (the abbreviation of Bilkent University). Each frame in the video displays the recorded intensity at any specified binding energy as an aerial map with the binding energy steps of $0.2 \mathrm{eV}$ in the range of $682-702 \mathrm{eV}$. The mapped area is ca. $5 \mathrm{~mm} \times 10 \mathrm{~mm}$ and each pixel corresponds to averaged data of $50 \mu \mathrm{m}$-diameter spot with steps of $50 \mu \mathrm{m}$ between data points. 